\title{
CD5 and CD6 as immunoregulatory biomarkers in non-small cell lung cancer
}

\author{
Andrea Moreno-Manuel ${ }^{1,2 \#}$, Eloisa Jantus-Lewintre ${ }^{1,2,3,4 \#}$, Ines Simões ${ }^{5}$, Fernando Aranda ${ }^{5}$, \\ Silvia Calabuig-Fariñas ${ }^{2,4,6}$, Esther Carreras $^{5}$, Sheila Zúñiga ${ }^{7}$, Yvonne Saenger ${ }^{8}$, Rafael Rosell ${ }^{9}$, \\ Carlos Camps $s^{1,2,4,10,11}$, Francisco Lozano ${ }^{5,12,13 *}$, Rafael Sirera ${ }^{1,2,3,4 *}$
}

${ }^{1}$ Molecular Oncology Laboratory, Fundación para la Investigación del Hospital General Universitario de Valencia, Valencia, Spain; ${ }^{2}$ TRIAL Mixed Unit, Centro de Investigación Príncipe Felipe-Fundación para la Investigación del Hospital General Universitario de Valencia, Valencia, Spain; ${ }^{3}$ Department of Biotechnology, Universitat Politècnica de València, Valencia, Spain; ${ }^{4}$ CIBERONC, Valencia, Spain; ${ }^{5}$ Immunoreceptors of the Innate and Adaptative System, Institut d'Investigacions Biomèdiques August Pi i Sunyer, Barcelona, Spain; ${ }^{6}$ Department of Pathology, Universitat de València, Valencia, Spain; ${ }^{7}$ Unidad de Medicina de Precisión en Oncología Traslacional, INCLIVA, Valencia, Spain; ${ }^{8}$ Department of Medicine, Columbia University College of Physicians and Surgeons, New York, NY, USA; ${ }^{9}$ Catalan Institute of Oncology, Germans Trias i Pujol Health Sciences Institute and Hospital, Badalona, Spain; ${ }^{10}$ Department of Medicine, Universitat de València, Valencia, Spain; ${ }^{11}$ Servicio de Oncología Médica, Consorcio Hospital General Universitario de Valencia, Valencia, Spain; ${ }^{12}$ Servei d'Immunologia, Centre de Diagnòstic Biomèdic, Hospital Clínic de Barcelona, Barcelona, Spain; ${ }^{13}$ Departament de Biomedicina, Facultat de Medicina, Universitat de Barcelona, Barcelona, Spain

Contributions: (I) Conception and design: E Jantus-Lewintre, C Camps, F Lozano, R Sirera; (II) Administrative support: None; (III) Provision of study materials or patients: None; (IV) Collection and assembly of data: A Moreno-Manuel, E Jantus-Lewintre, I Simões, F Aranda, S CalabuigFariñas, E Carreras, S Zúñiga, C Camps; (V) Data analysis and interpretation: A Moreno-Manuel, E Jantus-Lewintre, I Simões, F Aranda, Y Saenger, C Camps, R Rosell, F Lozano, R Sirera; (VI) Manuscript writing: All authors; (VII) Final approval of manuscript: All authors.

\#These authors contributed equally to this work.

*These authors are senior co-authors of this work.

Correspondence to: Rafael Sirera. Department of Biotechnology, Universitat Politècnica de València, Camino de Vera, s/n, 46022, Valencia, Spain. Email: rsirera@btc.upv.es; Francisco Lozano. Institut d'Investigacions Biomèdiques August Pi i Sunyer, Rossello 149-153, 08036-Barcelona, Spain. Email: flozano@clinic.cat.

Background: The study of immune surveillance in the tumour microenvironment is leading to the development of new biomarkers and therapies. The present research focuses on the expression of CD5 and CD6-two lymphocyte surface markers involved in the fine tuning of TCR signaling-as potential prognostic biomarkers in resectable stages of non-small cell lung cancer (NSCLC).

Methods: $C D 5$ and $C D 6$ gene expression was analysed by reverse transcription quantitative polymerase chain reaction (RTqPCR) in 186 paired fresh frozen tumour and normal tissue samples of resected NSCLC. Results: Patients with higher CDS expression had significantly increased overall survival (OS, 49.63 vs. 99.90 months, $\mathrm{P}=0.013)$. CD5 expression levels were correlated to CD4 infiltration and expression levels, and survival analysis showed that patients with a higher $C D 5 / C D 4^{+}$ratio had significantly improved prognosis. Multivariate analysis established CDS expression as an independent prognostic biomarker for OS in early stages of NSCLC (HR=0.554; 95\% CI, 0.360-0.853; P=0.007). Further survival analysis of NSCLC cases $(\mathrm{n}=97)$ from The Cancer Genome Atlas (TCGA) database, confirmed the prognostic value of both CD5 and CD6 expression, although CD6 expression alone did not reach significant prognostic value in our NSCLC training cohort.

Conclusions: Our data support further studies on CD5 and CD6 as novel prognostic markers in resectable NSCLC and other cancer types (i.e., melanoma), as well as a role for these receptors in immune surveillance.

Keywords: CD5; CD6; immune checkpoint; melanoma; non-small cell lung cancer (NSCLC)

Submitted Sep 27, 2019. Accepted for publication Feb 28, 2020.

doi: $10.21037 /$ tlcr-19-445

View this article at: http://dx.doi.org/10.21037/tlcr-19-445 


\section{Introduction}

The immune system's natural ability to detect and eliminate malignant cells is currently considered the best weapon in the battle against cancer. The study of immune biomarkers is critical to diagnose, prevent and choose the appropriate immunotherapy strategy in cancer treatment. The presence, localization, and proportion of helper and especially cytotoxic T cells (CTLs) in tumour-infiltrating lymphocytes (TILs) from aggressive cancers such as non-small cell lung cancer (NSCLC) and melanoma has been associated with a favorable prognosis (1-3). In recent years, gene expression patterns and single nucleotide polymorphism studies have contributed to cancer prognosis (4). Particularly, inducible immunoregulatory genes like immune checkpoints blockers (CTLA-4 or PD-1) expressed in TILs, are among the most predictive biomarkers for cancer clinical outcome and targets for immunotherapy $(5,6)$. Identification of new immune biomarkers is still needed to fully understand the mechanisms of immune evasion and facilitate subsequent development of novel immunotherapies.

The lymphocyte surface co-receptors CD5 and CD6 are immunomodulators involved in the development, activation, differentiation and survival of lymphocytes $(7,8)$. They are encoded by homologous genes derived from duplication of a common ancestral gene (9), and both are constitutively expressed by all $\mathrm{T}$ cells and the small B1a cell subset. CD5 and CD6 are signal-transducing receptors that physically associate with the $\mathrm{T}$ and $\mathrm{B}$ cell antigen-specific clonotypic receptor (TCR and BCR, respectively) at the centre of the immune synapse $(10,11)$. The endogenous CD6 ligands involve CD166/ALCAM (12), Galectins 1 and 3 (13) and CD318 (14), all broadly distributed on immune, epithelial, mesenchymal and/or cancer cells. In contrast, the nature of the CD5 ligand/s is ill-defined because no reported candidate $\left(\mathrm{CD} 72, \mathrm{IgV}_{\mathrm{H}}\right.$ framework region, gp200, gp150, gp40-80, CD5 itself and IL-6) has been firmly validated by independent groups $(7,15)$. Based on monoclonal antibody in vitro data, CD5 and CD6 were initially considered costimulatory molecules $(7,8)$. However, analysis of CD5 and CD6 knockout mice unveiled their negative modulatory role for thymocyte (and B1a) activation and differentiation signals upon clonotypic receptor cross-linking (16-19). Interestingly, this immunomodulatory role occurs even in the absence of ligand interaction $(20,21)$. This implies that lymphocyte function can be up- or down-regulated by $C D 5$ and $C D 6$ expression. Accordingly, anergic $\mathrm{T}$ and B cells show upregulated surface CD5 expression $(22,23)$.
CD5 and CD6 expression also parallels TCR/CD3 levels and is predictive of TCR avidity and survival of T cells (24-27). Moreover, regulation of CD5 expression by TCR engagement has been reported in peripheral $\mathrm{T}$ cells (28). Regarding antitumour responses, in situ regulation of $C D 5$ expression by CTLs is thought to adapt their sensitivity to intra-tumour peptide-major histocompatibility complex (p-MHC) levels (29). Indeed, CTL clones from lung cancer patients show that $C D 5$ expression is inversely proportional to their anti-tumour cytolytic activity, preventing activationinduced cell death (AICD) during $\mathrm{T}$ cell overactivation (29).

CD5 and CD6's modulation of lymphocyte activation and survival supports the hypothesis that their overall intra-tumour expression levels alter the anti-tumour immune response and can be therefore used as prognosis biomarkers in cancer. Accordingly, this study investigated the prognostic value of $C D 5$ and $C D 6$ gene expression in a cohort of 186 patients with resectable NSCLC. The results show that high intra-tumour levels of both CD5 and CD6 associate to better prognosis as measured by overall survival and relapse-free survival. This was validated in silico using NSCLC biopsy information from The Cancer Genome Atlas (TCGA) database.

\section{Methods}

\section{NSCLC patient cohort and sample collection}

The training cohort included 186 patients with resected and non-pretreated stage I to IIIA NSCLC from Consorcio Hospital General Universitario de Valencia. Between 2004 and 2017, 186 fresh-frozen tumour and normal tissue samples were obtained from surgical resection and preserved in RNAlater $^{\circledR}$ (Applied Biosystems, USA). Patients who had received neoadjuvant treatment and those with a followup shorter than 1 month were excluded. This study abides by the Declaration of Helsinki (as revised in 2013) and was approved by the institutional review board. All patients had signed the informed consent prior to sample collection.

\section{Real-time PCR analysis of NSCLC patients}

RNA was isolated using TRI Reagent ${ }^{\circledR}$ (Sigma, USA) and retrotranscribed using High Capacity cDNA Reverse Transcription Kit (Applied Biosystems, USA) in a MasterCycler ${ }^{\circledR}$ thermocycler (Eppendorf, Germany) following the manufacturer's instructions. $C D 3 D, C D 3 E$, $C D 4, C D 5, C D 6$ and $C D 8$ gene expression was analysed by 
RTqPCR using Taqman ${ }^{\circledR}$ hydrolysis probes and Taqman ${ }^{\circledR}$ Gene Expression Master Mix (Applied Biosystems, USA) in a LightCycler 480 thermocycler (Roche, Switzerland).

Relative gene expression was calculated by Pfaff formula, taking into consideration expression differences between normal and tumour tissue, as well as RTqPCR efficiencies of each TaqMan ${ }^{\circledR}$ assay. Reference gene expression corresponds to the geometric mean of $A C T B, C D K N 1 B$ and $G U S B$, endogenous controls used after evaluation with GeNorm software (30,31).

\section{Immunobistochemistry (IHC)}

$C D 5$ and $C D 6$ relative gene expression data were normalized against $\mathrm{CD}^{+}$and $\mathrm{CD} 8^{+}$lymphocyte infiltration in NSCLC samples $(n=60)$. To do so, CD4 and CD8 expression was evaluated in 60 Formalin-Fixed Paraffin Embedded (FFPE) samples using a Dako Autostainer Link 48 and the Dako EnVision ${ }^{\mathrm{TM}}$ FLEX detection system (Dako, Canada). After section drying and antigen dewaxing in a PT Link instrument, the endogenous peroxidase activity was quenched with peroxidase blocking reagent in the Autostainer Link 48 instrument. Immunostaining was carried out with Dako FLEX Ready to-Use format for CD4 (Clone 4B12, Dako) and CD8 (Clone C8/144B, Dako). Briefly, a detection system chromogen (3,3'-diaminobenzidine, DAB) was used after primary antibody incubation, followed by washing and counterstaining of sections with hematoxylin, dehydration and mounting. $\mathrm{CD} 4^{+}$and $\mathrm{CD} 8^{+}$lymphocytes were counted in 10 high power fields (HPF) (magnification $\times 400$ ) for tumour areas. Negative controls and normal human tonsil positive control tissue were included. IHC staining quantification was performed by two independent evaluators.

\section{TCGA database search for CD5 and CD6 mRNA expression levels in NSCLC biopsies}

Online information available at TCGA database (https:// cancergenome.nih.gov/) for NSCLC patients was downloaded and used as independent validation cohorts. Patients with resected NSCLC and available gene expression data for $C D 5$ and $C D 6$ in normal and tumour tissue samples were selected $(\mathrm{n}=97)$. Patients who had received neoadjuvant treatment or with follow-up shorter than 1 month were excluded.

\section{Statistical analyses}

Relative gene expression was dichotomized using median as a cut-off value. Non-parametric tests were used for correlations between clinico-pathological and analytical variables. Survival analyses were performed considering relapse-free survival (RFS) and overall survival (OS). RFS spans from surgery to relapse or exitus dates, and OS from surgery to exitus dates, following the Response Evaluation Criteria in Solid Tumours (RECIST). For patients who neither relapsed nor died, the last recorded followup was considered. Gene prognostic value was assessed using Kaplan-Meier curves (Log-rank test) and univariate Cox regression analyses, followed by a multivariate Cox regression analysis, using all significant variables to establish independent prognostic biomarkers. Statistical analyses were performed using the Statistical Package for Social Sciences (SPSS) 15.0 software (Chicago, USA), considering significant $\mathrm{P}<0.05$.

\section{Results}

\section{Analysis of CD5 and CD6 expression in NSCLC samples from training cohort}

The NSCLC training cohort was composed of 186 patients (see Table S1 for demographic and clinicopathological data), mainly men (85.0\%) and current or former smokers (88.7\%). Histology was squamous cell carcinoma in $47.9 \%$ and adenocarcinoma in $41.4 \%$ of all cases. During the followup (median 34.2 months), 85 patients relapsed (45.7\%) and 91 died $(48.9 \%)$. Non-parametric tests were conducted to determine an association of relative gene expression with clinicopathological variables.

The survival analyses revealed that high CD5 expression had significant biomarker prognostic value for OS (OS, 49.63 vs. 99.90 months, $\mathrm{P}=0.013)$. Furthermore, a statistical trend toward significant RFS was detected (RFS, 29.20 vs. 44.30 months, $\mathrm{P}=0.076$ ) (Table 1; Figure 1). In contrast, similar analyses did not provide significant results for CD6 expression (Table 1). Multivariate Cox regression analysis revealed high $C D 5$ expression as a potential prognostic biomarker for OS in resected NSCLC patients (HR=0.554; 95\% CI, 0.360-0.853; $\mathrm{P}=0.007$ ) (Table 2).

CD5 and CD6 are constitutively expressed lymphocytic receptors, whose expression can be regulated during lymphocyte development and activation events $(32,33)$. Thus, mRNA level correlation to lymphocyte infiltration 
Table 1 Survival analysis of CD5 and CD6 in NSCLC patients of training cohort. Cox univariate analyses were conducted with dichotomized relative expression of $C D 5$ and $C D 6$

\begin{tabular}{|c|c|c|c|c|c|c|}
\hline Univariate analysis & \multicolumn{3}{|c|}{ RFS } & \multicolumn{3}{|c|}{ OS } \\
\hline CD5 (high vs. low) & 0.708 & $0.483-1.038$ & 0.077 & 0.592 & $0.390-0.898$ & $0.014^{*}$ \\
\hline CD6 (high vs. low) & 0.997 & $0.680-1.460$ & 0.986 & 0.929 & $0.614-1.404$ & 0.725 \\
\hline
\end{tabular}

*, P value <0.05. NSCLC, non-small cell lung cancer; RFS, relapse-free survival; OS, overall survival; HR, hazard ratio; Cl, confidence interval.
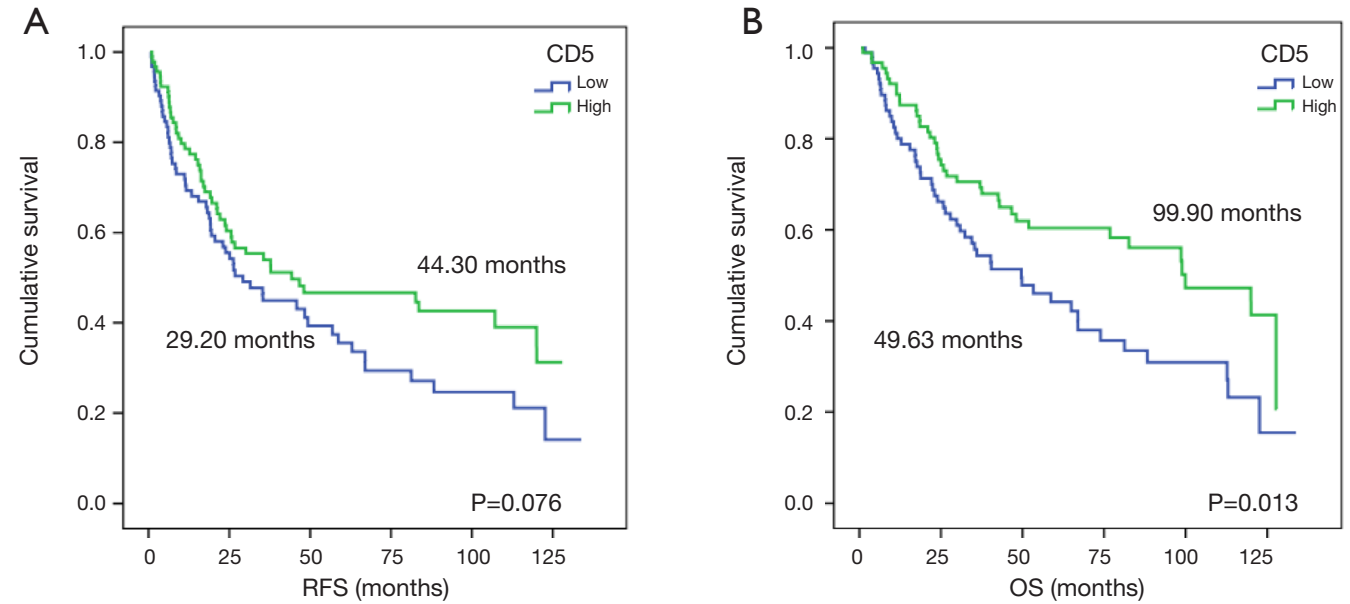

Figure 1 NSCLC survival curves according to CDS relative expression. Kaplan-Meier survival curves for (A) RFS and (B) OS of CD5 expression levels in NSCLC patients of training cohort. NSCLC, non-small cell lung cancer; RFS, relapse-free survival; OS, overall survival.

Table 2 Multivariate cox regression analysis in NSCLC patients of training cohort. Cox multivariate analysis was conducted with all significant variables resulted from the univariate analyses, that were performance status, stage, tumour size, lymph node involvement, KRAS mutational status and $C D 5$

\begin{tabular}{|c|c|c|c|c|c|c|}
\hline Multivariate analysis & \multicolumn{3}{|c|}{ RFS } & \multicolumn{3}{|c|}{ OS } \\
\hline Stage (IIIA vs. IIA/IIB vs. IA/IB) & 1.565 & $1.219-2.010$ & $0.0004^{*}$ & 1.625 & $1.234-2.139$ & $0.001^{*}$ \\
\hline KRAS mutational status (mutated vs. WT) & 2.126 & $1.280-3.531$ & $0.004^{*}$ & 2.054 & $1.134-3.723$ & $0.018^{*}$ \\
\hline PS (1/2 vs. 0) & 1.661 & $1.102-2.504$ & $0.015^{\star}$ & 1.800 & $1.148-2.823$ & $0.010^{*}$ \\
\hline
\end{tabular}

*, P value <0.05. NSCLC, non-small cell lung cancer; RFS, relapse-free survival; OS, overall survival; PS, performance status; WT, wildtype; HR, hazard ratio; $\mathrm{Cl}$, confidence interval.

or to up-regulation in infiltrating lymphocytes was assessed. To this end, $C D 5$ and $C D 6$ gene expression was normalized to $\mathrm{CD}^{+}$and $\mathrm{CD} 8^{+}$lymphocyte infiltration by IHC, and the $C D 5 / \mathrm{CD}^{+}, \mathrm{CD} 5 / \mathrm{CD}^{+}, \mathrm{CD} 6 / \mathrm{CD}^{+}$and $C D 6 / \mathrm{CD}^{+}$ratios were calculated in 60 samples. Survival analysis indicated that patients with higher $C D 5 / \mathrm{CD}^{+}$ratios had significantly improved prognosis (RFS, 13.33 vs. 66.97 months, $\mathrm{P}=0.023$; OS, 25.73 vs. 73.93 months, $\mathrm{P}=0.019$ ) (Figure S1). Furthermore, a tendency for improved RFS and OS was observed for $C D 5 / \mathrm{CD} 8^{+}, C D 6 / C D 4^{+}$and $C D 6 / C D 8^{+}$ratios 
Table 3 Survival analysis of CD5 and CD6 in NSCLC patients of TCGA database. Cox univariate analyses were conducted with dichotomized relative expression of $C D 5$ and $C D 6$

\begin{tabular}{lccccccc}
\hline \multirow{2}{*}{ Variables } & \multicolumn{4}{c}{ RFS } & & \multicolumn{2}{c}{ OS } \\
\cline { 2 - 3 } & HR & $95 \% ~ C l$ & P value & & HR & 95\% Cl & P value \\
\hline CD5 (high vs. low) & 0.542 & $0.306-0.960$ & $0.036^{*}$ & & 0.537 & $0.296-0.975$ & $0.041^{*}$ \\
CD6 (high vs. low) & 0.513 & $0.289-0.911$ & $0.023^{*}$ & & 0.530 & $0.292-0.961$ & $0.037^{*}$ \\
\hline
\end{tabular}

*, P value <0.05. RFS, relapse-free survival; NSCLC, non-small cell lung cancer; OS, overall survival; HR, hazard ratio; Cl, confidence interval.

\section{(Table S2).}

In order to confirm these results, relative $C D 5$ and $C D 6$ expression using $\mathrm{RTq} \mathrm{qCR}$ was normalized against $C D 4$ and CD8 expression in 186 NSCLC patients from our training cohort. Survival analysis confirmed that higher CD5/CD4 expression was associated to improved OS (OS, $49.80 v s$. 99.90 months, $\mathrm{P}=0.042$ ) (Figure S1), but no significant results were obtained neither for the rest of the ratios nor for RFS (Table S3). Additionally, and taking into account that the Spearman's test correlated $C D 5$ and $C D 6$ to $C D 3 D$ and $C D 3 E$, the $C D 5 / C D 3 D, C D 5 / C D 3 E, C D 6 / C D 3 D$ and $C D 6 / C D 3 E$ ratios were evaluated but showed no significant association to prognosis (Table S3).

\section{In silico analysis of CD 5 and CD6 expression from NSCLC TCGA database}

Online information of 97 NSCLC patients from the TCGA database was used as a validation cohort. Their median follow-up was 27.61 months, time in which $27(32.1 \%)$ patients had relapsed and 46 (47.4\%) were exitus. The statistical analysis confirmed that higher expression of both CD5 and CD6 improved prognosis in NSCLC patients, as it was associated with increased RFS (34.98 vs. 75.57 months, $\mathrm{P}=0.033 ; 25.31$ vs. 75.57 months, $\mathrm{P}=0.020$, respectively) and $\mathrm{OS}$ (40.49 vs. 77.97 months, $\mathrm{P}=0.038 ; 39.02$ vs. 77.97 months, $\mathrm{P}=0.034$, respectively) (Table 3; Figure 2). Multivariate analysis did not provide significant results on account of the small sample size $(\mathrm{n}=97)$. In all, our data supports that higher CD5 expression in early-stage NSCLC patients associates to increased OS. Regarding CD6, the validation cohort indicates a NSCLC biomarker potential that should be explored further.

\section{Discussion}

The presence of immune cells in the tumour microenvironment plays a key role in NSCLC and melanoma prognosis $(34,35)$. Our work provides a prognostic value for intratumour CD5 and CD6 mRNA expression, two lymphocyte co-receptors involved in modulation of $\mathrm{T}$ (and B1a) cell development, activation, differentiation and survival $(7,8)$. High $C D 5$ expression associates to favourable prognosis in 186 fresh-frozen samples of a NSCLC training cohort, validated further with online data from the NSCLC TCGA database. The analysis of CD6 expression in NSCLC tumour samples reveals similar associations. Furthermore, $C D 5$ and $C D 6$ expression correlate to the number of $\mathrm{CD} 4^{+}$and $\mathrm{CD} 8^{+}$lymphocytes and these ratios indicate better prognosis. Importantly, better prognosis also correlates with $C D 5$ overexpression by TILs.

In order to explore the prognostic potential of $C D 5$ and $C D 6$ expression in other cancer types, melanoma information from the TCGA database was analyzed. As illustrated in Figure $S 2$, better survival rate ("alive" group) presented significantly higher $C D 5$ and $C D 6$ expression, together with other $\mathrm{T}$ cell $(C D 3, C D 4, C D 8)$ and $\mathrm{B}$ cell (CD19) markers. There was also a significant overall difference rate between the "alive" and "dead" groups regarding time to progression (TTP) and OS considering high vs. low $C D 5$ (Figure $S 3 A, B$ ) or $C D 6$ expression (Figure $S 3 C, D)$.

In addition, patients with better survival and tumour regression (no tumour in last follow-up) showed higher $C D 5$ and $C D 6$ expression, but lower CD3, CD4, CD8, CD19 levels (Figure $S 2 B$ ), in support of infiltrating lymphocytes over-expressing $C D 5$ and $C D 6$ with better prognosis in melanoma.

Tumour microenvironment genetics has identified inhibitory or stimulatory lymphocyte accessory molecules (e.g., PD-1/CD279, CTLA-4, LAG-3, TIM-3 and 4-1BB/ CD137) that can be regulated to modulate T-cell activation and survival. Interestingly, these biomarkers distinguish tumour-specific $\mathrm{T}$ cells from unrelated $\mathrm{T}$ cells in the tumour infiltrate $(36,37)$. This is illustrated by PD-1- 

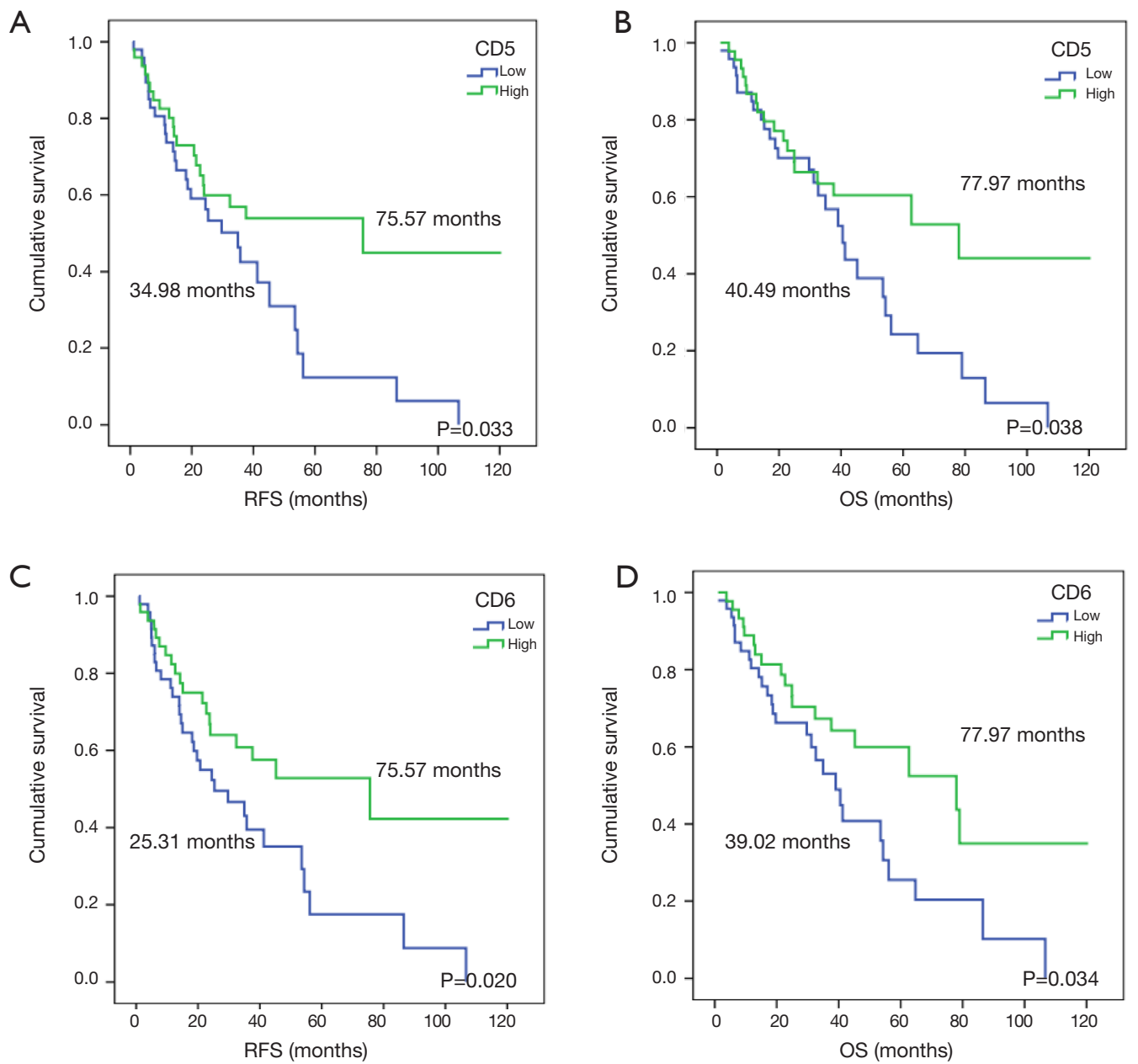

Figure 2 NSCLC survival curves according to the expression status of CD5 and CD6 of TCGA database patients. Kaplan-Meier survival curves for RFS and OS of CD5 (A and B) CD6 (C and D) expression levels in NSCLC patients of TCGA database. NSCLC, non-small cell lung cancer; RFS, relapse-free survival; OS, overall survival.

an inhibitory receptor inducibly expressed on activated $\mathrm{T}$ cells-as a marker of the tumour-reactive $\mathrm{CD}^{+} \mathrm{T}$ cell fraction in melanoma tumours (37) and of high avidity $\mathrm{CD}^{+} \mathrm{T}$ cells specific for Melan-A (38) or neoantigens (39). PD-1 expression is related to TCR signal strength, and thus to the functional avidity of specific $\mathrm{T}$ cells, underlining the complex significance of PD-1 expression on tumour-specific T cells (38).

Similar to PD-1 and CTLA-4, the CD5 and CD6 coreceptors act as negative regulators of $\mathrm{T}$ cell activation $(8,40)$. CD5- and CD6-deficient thymocytes are hyperreactive to TCR/CD3 cross-linking $(16,18)$. Surface CD5 and CD6 expression is set in the thymus, and parallels TCR/ CD3, becoming predictive of TCR avidity $(16,18,19,24,25)$. Thus, thymocytes binding self-peptide-MHC (self-pMHC) with high affinity consequently deliver strong TCRmediated activation signals, and express higher CD5 and CD6 surface levels to overcome negative selection. Surface CD5 and CD6 promote thymocyte survival by different mechanisms $(25,41)$. Post-positively selected (peripheral) $\mathrm{CD}^{+}$and $\mathrm{CD} 8^{+} \mathrm{T}$ cells with high CD5/CD6 expression $\left(\mathrm{CD} 5 / 6^{\mathrm{hi}}\right)$ respond to foreign peptides with increased activation and survival (42-44). In other words, $T$ cells with TCRs of stronger avidity for self-pMHC dominate in responses to foreign antigens and accumulate in aging individuals, revealing that positive selection contributes to effective immunity (42). This would be in line with our finding that high intratumour CD5 (and CD6) expression correlates with better NSCLC and Melanoma prognosis. Accordingly, higher intratumour CD5 (and CD6) levels 
reflect infiltrating $\mathrm{T}$ cells with higher avidity for tumour antigens and more resistant to activation-induced cell death (29).

Self-pMHC contact modulates $C D 5$ expression, survival and homeostatic proliferation of naïve $\mathrm{T}$ cells in the periphery $(28,45)$ suggesting modulation of CD 5 on TILs at the tumour site. Indeed, in situ CD5 expression adaptation to $\mathrm{pMHC}$ levels of TILs from a lung carcinoma patient has been reported (46). The decreased MHC class I expression, observed in tumour escape from $\mathrm{CD}^{+} \mathrm{T}$-cell killing, would induce TILs down-modulation of CDS to prevent tumour evasion. In line with this, $\mathrm{CD} 5^{\text {lo }} \mathrm{CTL}$ clones from lung carcinoma patients displayed higher tumour-specific cytotoxicity than $\mathrm{CD} 5^{\text {hi }}$ clones and increased susceptibility to tumour-induced AICD (29). This is in agreement with CD5's prevention of AICD by negatively regulating T-cell activation (47). Higher AICD susceptibility of CD $5^{\text {lo }}$ CTLs would explain the transient control of tumour growth observed in CD5-deficient mice challenged with melanoma (48). Our work demonstrates that protection of CD5-deficient $T$ cells from AICD by adenoviral-mediated expression of soluble Fas-Fc results in reduced melanoma growth (48). The latter may reflect a therapeutic strategy for patients showing low intratumour CD5 mRNA levels. In contrast, potentiation of TILs efficiency in tumours with high CD5 expression should include CD5 and Fas-FasL blocking strategies. A necessary CD5 blockade is supported by improved anti-tumour responses in mice expressing the soluble human CD5 (shCD5) transgene or injected with recombinant shCD5 $(49,50)$.

Recent IHC analyses in small series $(n=30)$ of untreated advanced-stage NSCLC patients concluded that intratumour high $\mathrm{CD}^{+}$and low $\mathrm{CD}^{+}$infiltrates associate to poor prognosis (51). Melanoma patient genetics has revealed that the hypofunctional CD 5 haplotype (isoform Pro224-Ala471, a poor down-regulator of TCR/CD3mediated T-cell activation) associates to better survival (29).

This is the first study of CD6 expression in tumourresected samples from early-stage NSCLC patients. No significance has been obtained in our NSCLC training cohort, but higher CD6 expression correlates with improved RFS and OS in our analyses of NSCLC and melanoma TCGA data. There is little information on the function of CD6. However, the homology between $C D 5$ and $C D 6$, may enable some degree of functional redundancy. Knockout mice show that both receptors share modulatory roles in thymocyte activation (negative) and survival (positive) $(16,18,19)$. Moreover, Nur-77 levels -indicative of TCR signaling strength- are elevated in $\mathrm{CD} 6^{\mathrm{hi}}$ compared to $\mathrm{CD} 6^{\text {lo }}$ peripheral $\mathrm{T}$ cells $(18) . \mathrm{CD} 6^{\mathrm{lo} / \text { neg }}$ peripheral $\mathrm{T}$ cell populations are less responsive to T-cell activators, more susceptible to apoptosis and enriched in regulatory $\mathrm{T}$ cells (Treg) $(27,52)$. High TCR avidity and survival of CD6 ${ }^{\text {hi }}$ $\mathrm{T}$ cells would be compatible with high intratumour $C D 6$ expression and favourable cancer prognosis, suggesting its biomarker potential only awaits confirmation.

In order to determine if $C D 5$ expression association to favourable prognosis is due to higher lymphocyte infiltration, $C D 5$ and $C D 6$ ratios to $C D 4$ and $C D 8$ were calculated. Our results confirm their prognostic value and support that higher $C D 5$ expression and lymphocyte infiltration associate to increased antitumour immune responses and improved patient prognosis in early-stage NSCLC.

\section{Conclusions}

This study points to a positive prognostic role for two lymphocyte inhibitory co-receptors, $C D 5$ and $C D 6$, in early-stage NSCLC (and in Melanoma). This conclusion is compatible with high surface levels of both CD5 and CD6 associated to TCR avidity and resistance to AICD. This evidence suggests that CD5 and CD6, along with other checkpoint inhibitors (e.g., PD-1 and CTLA-4), may be additional markers of tumour-specific T cells. Further studies deciphering the exact role of CD5, CD6 and their ligands in cancer would benefit patient stratification for personalized immunotherapies and development of new and more efficient strategies.

\section{Acknowledgments}

The authors thank Marcos Isamat for critical edition of the manuscript.

Funding: This work was supported by Spanish Health Institute Carlos III (ISCII, Fondo de Investigación Sanitaria; PI15-00753 to RS and P118/00226 to EJ and CC), Spanish Ministry of Economy, Industry and Competitiveness (MINECO, Plan Nacional de I+D+i; SAF2016-80535-R to FL) -co-financed by European Development Regional Fund "A way to achieve Europe" ERDF; Worldwide Cancer Research (14-1275 to FL), Fundació La Marató TV3 (201319-30-31-32 to FL and YS), and Agència de Gestió d'Ajuts Universitaris i de Recerca (AGAUR) from Generalitat de Catalunya (2017/SGR/1582 to FL). AM is recipient of a $\mathrm{PhD}$ scholarship from Asociación Española 
Contra el Cáncer (AECC) Scientific Foundation and Junta Provincial Asociada de Valencia AECC; IS, FA and EC are recipients of fellowships from Fundação para a Ciência e a Tecnologia (SFRH/ BD/75738/2011), Sara Borrell Program from ISCIII (CD15/00016), and European Community Seventh Framework Program (BIOTRACK, FP7/2007/2013; 229673), respectively.

\section{Footnote}

Conflicts of Interest: All authors have completed the ICMJE uniform disclosure form (available at http://dx.doi. org/10.21037/tlcr-19-445). RR serves as an unpaid editorial board member of Translational Lung Cancer Research from Jun 2019 to May 2022. The other authors have no conflicts of interest to declare.

Ethical Statement: The authors are accountable for all aspects of the work in ensuring that questions related to the accuracy or integrity of any part of the work are appropriately investigated and resolved. The study was approved by institutional ethics board of Hospital General Universitario de Valencia (Approval date: $28^{\text {th }}$ May 2015). The study was conducted in accordance with the Declaration of Helsinki (as revised in 2013) and all patients had signed the informed consent prior to the collection of their biological samples.

Open Access Statement: This is an Open Access article distributed in accordance with the Creative Commons Attribution-NonCommercial-NoDerivs 4.0 International License (CC BY-NC-ND 4.0), which permits the noncommercial replication and distribution of the article with the strict proviso that no changes or edits are made and the original work is properly cited (including links to both the formal publication through the relevant DOI and the license). See: https://creativecommons.org/licenses/by-nc-nd/4.0/.

\section{References}

1. Fortes C, Mastroeni S, Mannooranparampil TJ, et al. Tumor-infiltrating lymphocytes predict cutaneous melanoma survival. Melanoma Res 2015;25:306-11.

2. Reynders K, De Ruysscher D. Tumor infiltrating lymphocytes in lung cancer: a new prognostic parameter. J Thorac Dis 2016;8:E833-5.

3. Lee N, Zakka LR, Mihm MC, et al. Tumour-infiltrating lymphocytes in melanoma prognosis and cancer immunotherapy. Pathology 2016;48:177-87.

4. Baxevanis CN, Anastasopoulou EA, Voutsas IF, et al. Immune biomarkers: how well do they serve prognosis in human cancers? Expert Rev Mol Diagn 2015;15:49-59.

5. Kerr KM, Tsao MS, Nicholson AG, et al. Programmed Death-Ligand 1 Immunohistochemistry in Lung Cancer: In what state is this art? J Thorac Oncol 2015;10:985-9.

6. Usó M, Jantus-Lewintre E, Bremnes RM, et al. Analysis of the immune microenvironment in resected non-small cell lung cancer: the prognostic value of different $T$ lymphocyte markers. Oncotarget 2016;7:52849-61.

7. Consuegra-Fernández M, Aranda F, Simões I, et al. CD5 as a Target for Immune-Based Therapies. Crit Rev Immunol 2015;35:85-115.

8. Santos RF, Oliveira L, Carmo AM. Tuning T Cell Activation: The Function of CD6 At the Immunological Synapse and in T Cell Responses. Curr Drug Targets 2016;17:630-9.

9. Lecomte O, Bock JB, Birren BW, et al. Molecular linkage of the mouse CD5 and CD6 genes. Immunogenetics 1996;44:385-90.

10. Brossard C, Semichon M, Trautmann A, et al. CD5 inhibits signaling at the immunological synapse without impairing its formation. J Immunol 2003;170:4623-9.

11. Gimferrer I, Calvo M, Mittelbrunn M, et al. Relevance of CD6-mediated interactions in T cell activation and proliferation. J Immunol 2004;173:2262-70.

12. Bowen MA, Patel DD, Li X, et al. Cloning, mapping, and characterization of activated leukocyte-cell adhesion molecule (ALCAM), a CD6 ligand. J Exp Med 1995; 181:2213-20.

13. Escoda-Ferran C, Carrasco E, Caballero-Baños M, et al. Modulation of CD6 function through interaction with Galectin-1 and -3. FEBS Lett 2014;588:2805-13.

14. Enyindah-Asonye G, Li Y, Ruth JH, et al. CD318 is a ligand for CD6. Proc Natl Acad Sci 2017;114:E6912-21.

15. Masuda K, Ripley B, Nyati KK, et al. Arid5a regulates naive CD4 + T cell fate through selective stabilization of Stat3 mRNA. J Exp Med 2016;213:605-19.

16. Tarakhovsky A, Kanner SB, Hombach J, et al. A role for CD5 in TCR-mediated signal transduction and thymocyte selection. Science 1995;269:535-7.

17. Bikah G, Carey J, Ciallella JR, et al. CD5-mediated negative regulation of antigen receptor-induced growth signals in B-1 B cells. Science 1996;274:1906-9.

18. Orta-Mascaró $M$, Consuegra-Fernández $M$, Carreras $E$, et al. CD6 modulates thymocyte selection and peripheral T cell homeostasis. J Exp Med 2016;213:1387-97. 
19. Li Y, Singer NG, Whitbred J, et al. CD6 as a potential target for treating multiple sclerosis. Proc Natl Acad Sci U S A 2017;114:2687-92.

20. Bhandoola A, Bosselut R, Yu Q, et al. CD5-mediated inhibition of TCR signaling during intrathymic selection and development does not require the CD5 extracellular domain. Eur J Immunol 2002;32:1811-7.

21. Oliveira MI, Gonçalves CM, Pinto M, et al. CD6 attenuates early and late signaling events, setting thresholds for T-cell activation. Eur J Immunol 2012;42:195-205.

22. Stamou P, de Jersey J, Carmignac D, et al. Chronic exposure to low levels of antigen in the periphery causes reversible functional impairment correlating with changes in CD5 levels in monoclonal CD8 T cells. J Immunol 2003;171:1278-84.

23. Hippen KL, Tze LE, Behrens TW. CD5 maintains tolerance in anergic B cells. J Exp Med 2000;191:883-90.

24. Azzam HS, Grinberg A, Lui K, et al. CD5 expression is developmentally regulated by $\mathrm{T}$ cell receptor (TCR) signals and TCR avidity. J Exp Med 1998;188:2301-11.

25. Singer NG, Fox DA, Haqqi TM, et al. CD6: expression during development, apoptosis and selection of human and mouse thymocytes. Int Immunol 2002;14:585-97.

26. Kassiotis G, Zamoyska R, Stockinger B. Involvement of Avidity for Major Histocompatibility Complex in Homeostasis of Naive and Memory T Cells. J Exp Med 2003;197:1007-16.

27. Carrasco E, Escoda-Ferran C, Climent N, et al. Human CD6 Down-Modulation following T-Cell Activation Compromises Lymphocyte Survival and Proliferative Responses. Front Immunol 2017;8:769.

28. Smith K, Seddon B, Purbhoo MA, et al. Sensory adaptation in naive peripheral CD4 T cells. J Exp Med 2001;194:1253-61.

29. Tabbekh M, Mokrani-Hammani M, Bismuth G, et al. T-cell modulatory properties of CD5 and its role in antitumor immune responses. Oncoimmunology 2013;2:e22841.

30. Pfaffl MW. A new mathematical model for relative quantification in real-time RT-PCR. Nucleic Acids Res 2001;29:e45.

31. Vandesompele J, De Preter K, Pattyn F, et al. Accurate normalization of real-time quantitative RT-PCR data by geometric averaging of multiple internal control genes. Genome Biol 2002;3:research0034.1-research0034.11.

32. Arman M, Aguilera-Montilla N, Mas V, et al. The human CD6 gene is transcriptionally regulated by RUNX and Ets transcription factors in T cells. Mol Immunol
2009;46:2226-35.

33. Arman M, Calvo J, Trojanowska ME, et al. Transcriptional Regulation of Human CD5: Important Role of Ets Transcription Factors in CD5 Expression in T Cells. J Immunol 2004;172:7519-29.

34. Thomas NE, Busam KJ, From L, et al. TumorInfiltrating Lymphocyte Grade in Primary Melanomas Is Independently Associated With Melanoma-Specific Survival in the Population-Based Genes, Environment and Melanoma Study. J Clin Oncol 2013;31:4252-9.

35. Usó M, Jantus-Lewintre E, Calabuig-Fariñas S, et al. Analysis of the prognostic role of an immune checkpoint score in resected non-small cell lung cancer patients. Oncoimmunology 2016;6:e1260214.

36. $\mathrm{Pu} \mathrm{X}$, Hildebrandt MAT, Lu C, et al. InflammationRelated Genetic Variations and Survival in Patients With Advanced Non-Small Cell Lung Cancer Receiving FirstLine Chemotherapy. Clin Pharmacol Ther 2014;96:360-9.

37. Gros A, Robbins PF, Yao X, et al. PD-1 identifies the patient-specific CD8+ tumor-reactive repertoire infiltrating human tumors. J Clin Invest 2014;124:2246-59.

38. Simon S, Vignard V, Florenceau L, et al. PD-1 expression conditions $\mathrm{T}$ cell avidity within an antigen-specific repertoire. Oncoimmunology 2015;5:e1104448.

39. Gros A, Parkhurst MR, Tran E, et al. Prospective identification of neoantigen-specific lymphocytes in the peripheral blood of melanoma patients. Nat Med 2016;22:433-8.

40. Freitas CM, Johnson D, Weber K. T Cell Calcium Signaling Regulation by the Co-Receptor CD5. Int J Mol Sci 2018;19:1295.

41. Mier-Aguilar CA, Cashman KS, Raman C, et al. CD5-CK2 Signaling Modulates Erk Activation and Thymocyte Survival. Baldwin TA, editor. PLoS One 2016;11:e0168155.

42. Mandl JN, Monteiro JP, Vrisekoop N, et al. T cell-positive selection uses self-ligand binding strength to optimize repertoire recognition of foreign antigens. Immunity 2013;38:263-74.

43. Persaud SP, Parker CR, Lo WL, et al. Intrinsic CD4+ $\mathrm{T}$ cell sensitivity and response to a pathogen are set and sustained by avidity for thymic and peripheral complexes of self peptide and MHC. Nat Immunol 2014;15:266-74.

44. Fulton RB, Hamilton SE, Xing Y, et al. The TCR's sensitivity to self peptide-MHC dictates the ability of naive CD8+ $\mathrm{T}$ cells to respond to foreign antigens. Nat Immunol 2015;16:107-17.

45. Ernst B, Lee DS, Chang JM, et al. The peptide ligands 
mediating positive selection in the thymus control $\mathrm{T}$ cell survival and homeostatic proliferation in the periphery. Immunity 1999;11:173-81.

46. Dorothée G, Vergnon I, El Hage F, et al. In situ sensory adaptation of tumor-infiltrating $\mathrm{T}$ lymphocytes to peptideMHC levels elicits strong antitumor reactivity. J Immunol 2005;174:6888-97.

47. Axtell RC, Xu L, Barnum SR, et al. CD5-CK2 binding/ activation-deficient mice are resistant to experimental autoimmune encephalomyelitis: protection is associated with diminished populations of IL-17-expressing T cells in the central nervous system. J Immunol 2006;177:8542-9.

48. Tabbekh M, Franciszkiewicz K, Haouas H, et al. Rescue of Tumor-Infiltrating Lymphocytes from Activation-Induced Cell Death Enhances the Antitumor CTL Response in CD5-Deficient Mice. J Immunol 2011;187:102-9.

Cite this article as: Moreno-Manuel A, Jantus-Lewintre E, Simões I, Aranda F, Calabuig-Fariñas S, Carreras E, Zúñiga S, Saenger Y, Rosell R, Camps C, Lozano F, Sirera R. CD5 and CD6 as immunoregulatory biomarkers in non-small cell lung cancer. Transl Lung Cancer Res 2020;9(4):1074-1083. doi: 10.21037/tlcr-19-445
49. Fenutría R, Martinez VG, Simões I, et al. Transgenic expression of soluble human CD5 enhances experimentally-induced autoimmune and anti-tumoral immune responses. PLoS One 2014;9:e84895.

50. Simões IT, Aranda F, Carreras E, et al. Immunomodulatory effects of soluble CD5 on experimental tumor models. Oncotarget 2017;8:108156-69.

51. Dirican N, Karakaya YA, Günes S, et al. Association of Intratumoral Tumor Infiltrating Lymphocytes and Neutrophil-to- Lymphocyte Ratio Are an Independent Prognostic Factor in Non-Small Cell Lung Cancer. Clin Respir J 2017;11:789-96.

52. Garcia Santana CA, Tung JW, Gulnik S. Human treg cells are characterized by low/negative CD6 expression. Cytometry A 2014;85:901-8. 

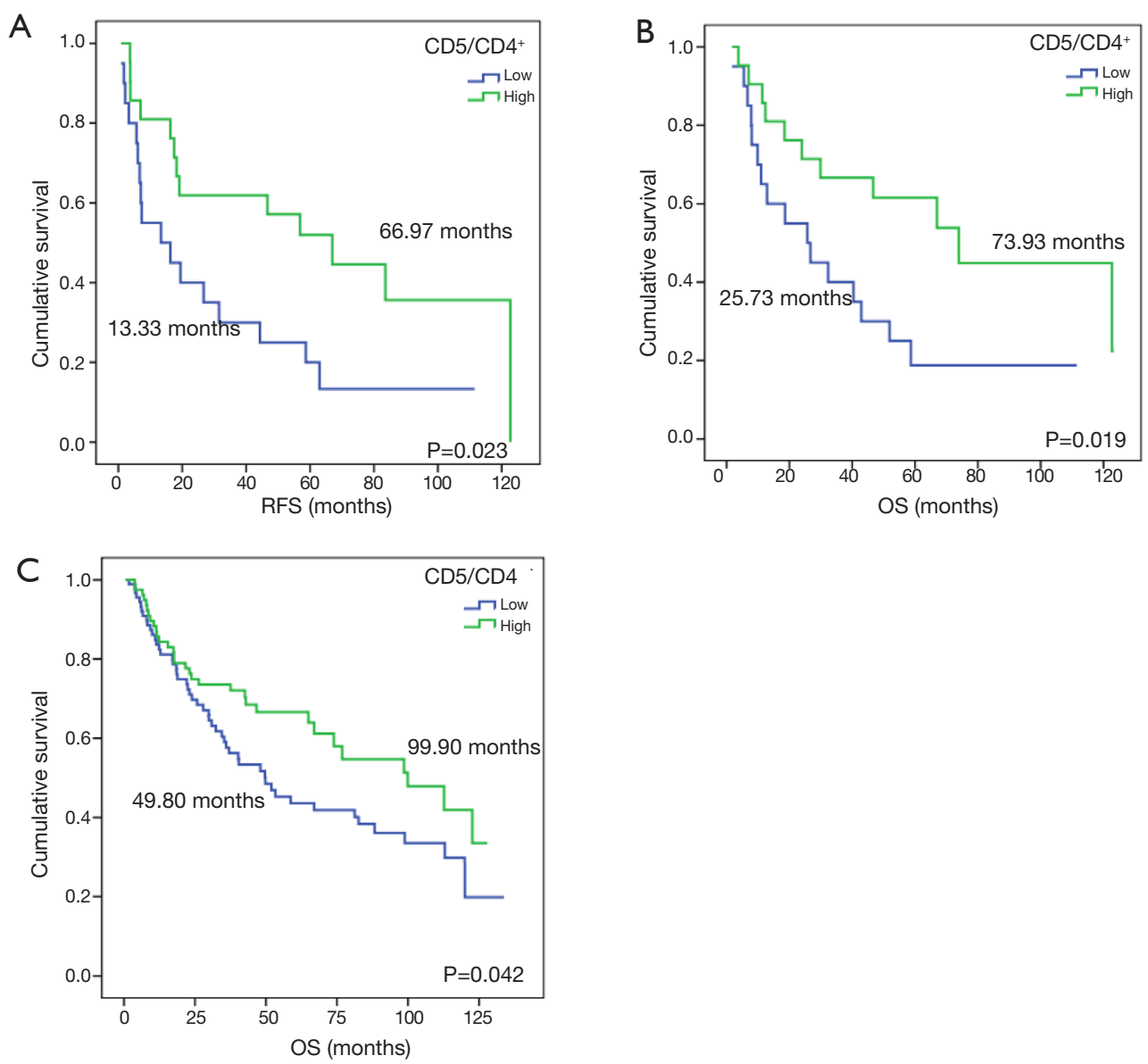

Figure S1 NSCLC survival curves according to CD5 ratios. (A) Kaplan-Meier survival curve for RFS of CD5 relative expression normalized to $\mathrm{CD} 4$ infiltration $\left(\mathrm{CD} 5 / \mathrm{CD} 4{ }^{+}\right)$. (B) Kaplan-Meier survival curve for OS of CD5 relative expression normalized to CD4 infiltration (CD5/ $\mathrm{CD}^{+}$). (C) Kaplan-Meier survival curve for OS of CD5/CD4 expression levels in NSCLC patients of the training cohort. NSCLC, nonsmall cell lung cancer; RFS, relapse-free survival; OS, overall survival. 

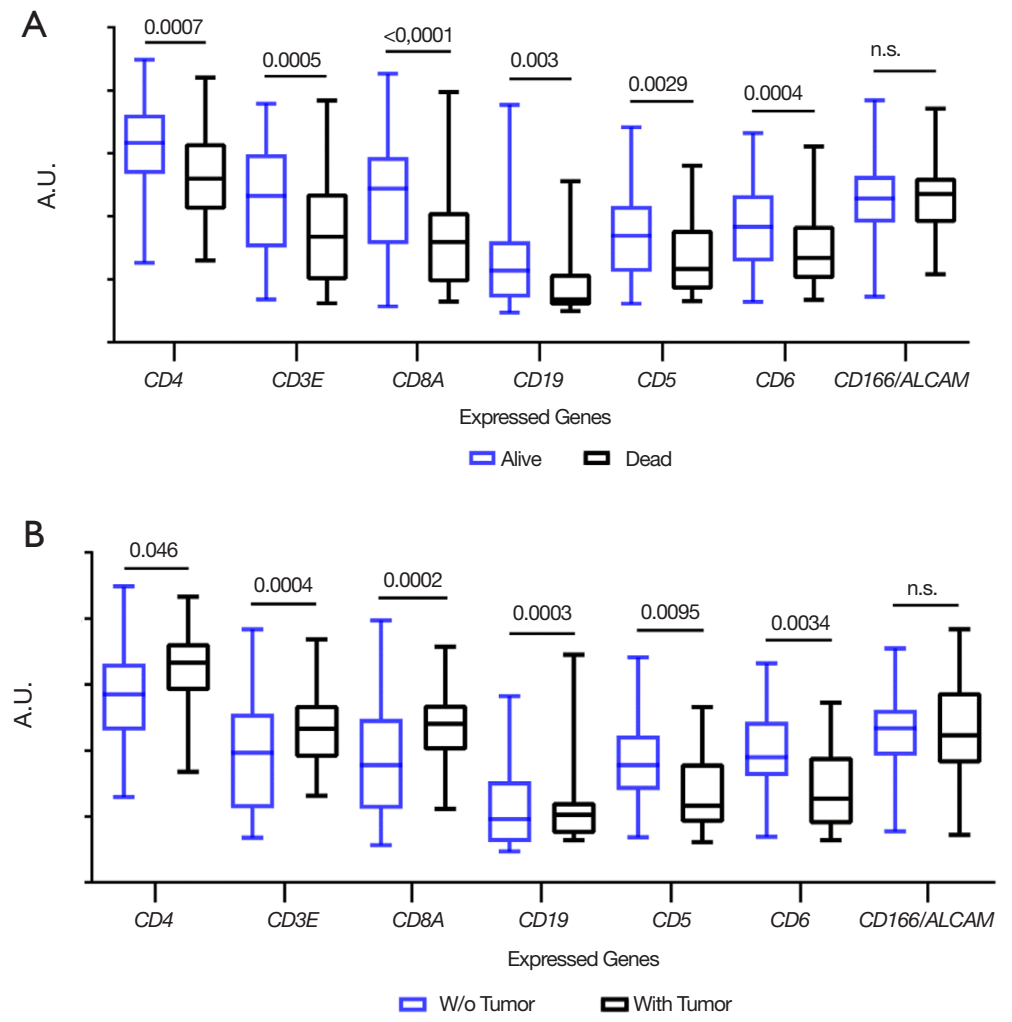

Figure S2 Retrospective analysis of gene expression in human melanoma samples from TCGA database. (A) Melanoma tumor biopsies ( $\mathrm{n}=409)$ were divided into "alive" [being alive for more than 4 years (1,460 days) from day of diagnosis; $\mathrm{n}=74$, blue plot] or "dead" [being dead in less than 2 years (730 days) from diagnosis; $\mathrm{n}=49$ ], black plot] groups. (B) The alive group was subdivided into two other groups; with tumor (information of last follow-up; $\mathrm{n}=16$, black plot) and without tumor (information of last follow-up; $\mathrm{n}=58$, blue plot). Gene expression values of $C D 3 \delta, C D 3 \varepsilon, C D 4, C D 8 \alpha, C D 8 \beta, C D 19, C D 6, C D 5$ and $A L C A M$ are presented in both graphs. Mann-Whitney test was used for statistical comparisons between groups. 
A
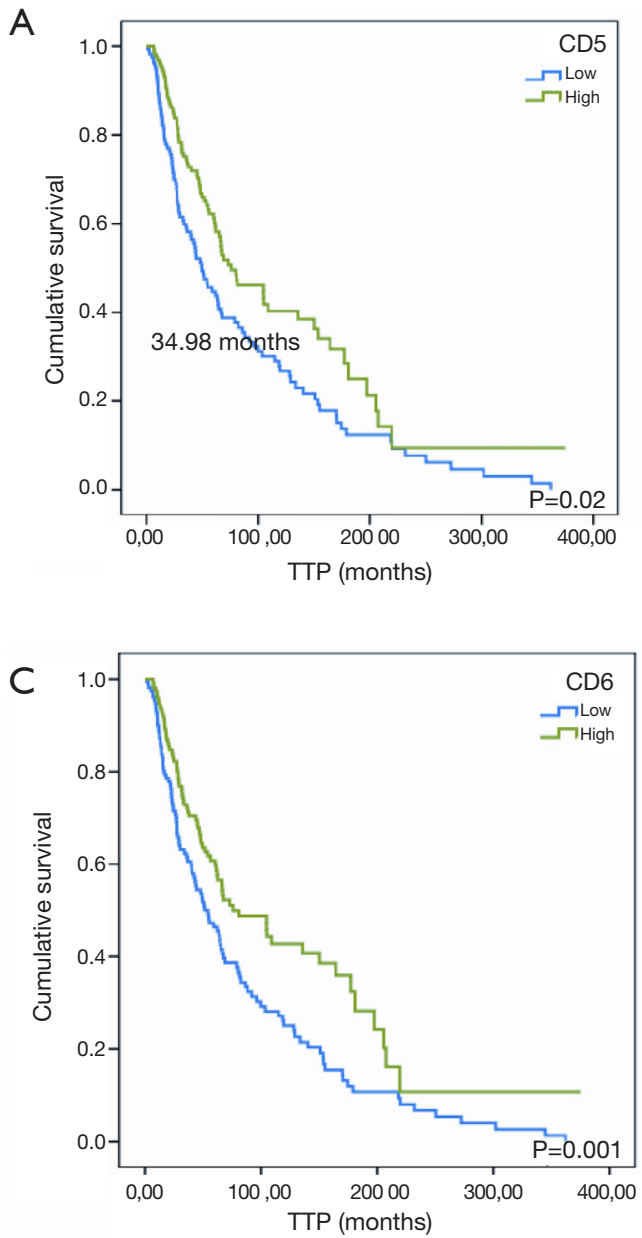

B
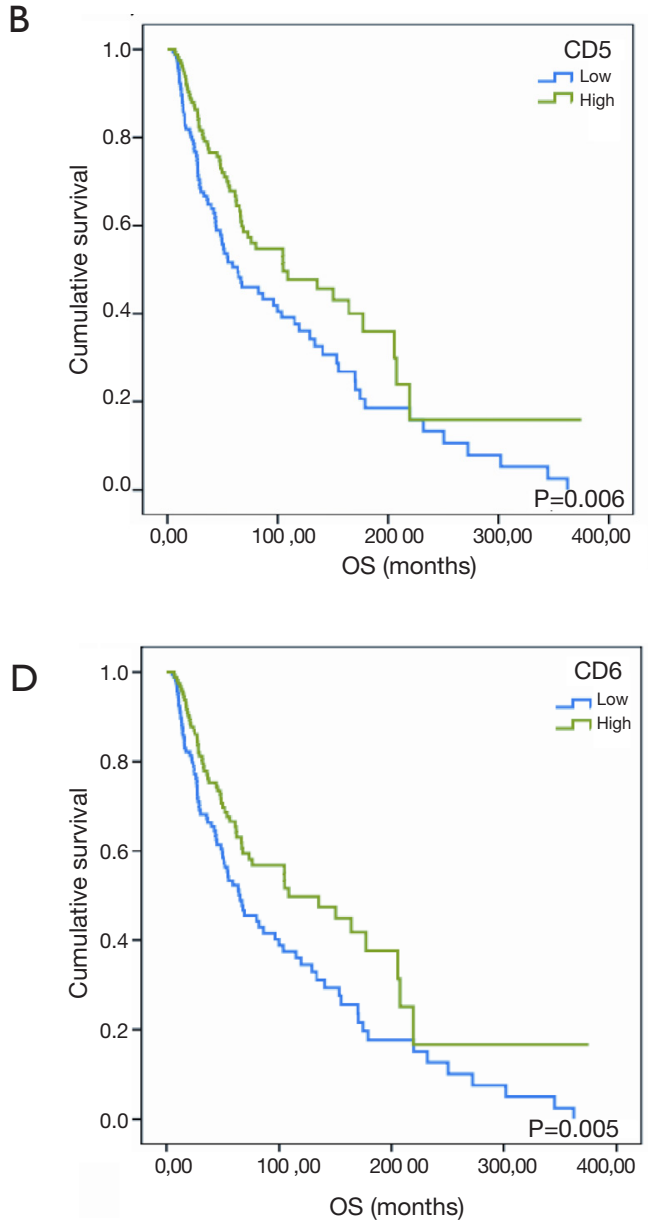

Figure S3 Melanoma-specific survival curves according to the expression status of $C D 5$ and $C D 6$. Kaplan-Meier survival curves for time to progression (TTP) and OS of high vs. low CD5 (A and B) or CD6 (C and D) expression levels, in tumor samples from melanoma patients. OS, overall survival. 
Table S1 Clinicopathological characteristics of patients included in NSCLC training Cohort

\begin{tabular}{|c|c|c|}
\hline Characteristics & $\mathrm{N}, \mathrm{n}=186$ & $\%$ \\
\hline Age at surgery (median, range) & \multicolumn{2}{|c|}{65 [26-85] } \\
\hline \multicolumn{3}{|l|}{ Gender } \\
\hline Male & 158 & 84.95 \\
\hline Female & 28 & 15.05 \\
\hline \multicolumn{3}{|l|}{ Smoking Status } \\
\hline Current & 91 & 48.92 \\
\hline Former & 74 & 39.79 \\
\hline Never & 21 & 11.29 \\
\hline \multicolumn{3}{|l|}{ Performance Status } \\
\hline 0 & 125 & 67.20 \\
\hline $1-2$ & 61 & 32.80 \\
\hline \multicolumn{3}{|l|}{ Stage } \\
\hline I & 96 & 51.61 \\
\hline II & 55 & 29.57 \\
\hline IIIA & 35 & 18.82 \\
\hline \multicolumn{3}{|l|}{ Lymph node involvement } \\
\hline Yes & 52 & 27.96 \\
\hline No & 134 & 72.04 \\
\hline \multicolumn{3}{|l|}{ Histology } \\
\hline SCC & 89 & 47.85 \\
\hline ADC & 77 & 41.40 \\
\hline Others & 20 & 10.75 \\
\hline \multicolumn{3}{|l|}{ Differentiation grade } \\
\hline Poor & 47 & 30.52 \\
\hline Moderate & 73 & 47.40 \\
\hline Well & 34 & 22.08 \\
\hline NS & 32 & \\
\hline \multicolumn{3}{|l|}{ EGFR } \\
\hline Wild type & 89 & 89.00 \\
\hline Mutated & 11 & 11.0 \\
\hline NS & 86 & \\
\hline \multicolumn{3}{|l|}{ KRAS } \\
\hline Wild type & 152 & 83.98 \\
\hline Mutated & 29 & 16.02 \\
\hline NS & 5 & \\
\hline \multicolumn{3}{|l|}{ Relapse } \\
\hline Yes & 85 & 45.70 \\
\hline No & 101 & 54.30 \\
\hline \multicolumn{3}{|l|}{ Exitus } \\
\hline Yes & 91 & 48.92 \\
\hline No & 95 & 51.08 \\
\hline
\end{tabular}

NSCLC, non-small cell lung cancer; SCC, squamous cell carcinoma; ADC, adenocarcinoma; NS, not specified. 
Table S2 Survival analysis of $C D 5$ and $C D 6$ ratios in NSCLC patients of training cohort. Cox univariate analyses were conducted with dichotomized relative expression of $C D 5$ and $C D 6$, normalized against $\mathrm{CD}^{+}{ }^{+}$and $\mathrm{CD} 8^{+}$infiltration measured by $\mathrm{IHC}$ in a subset of 60 patients

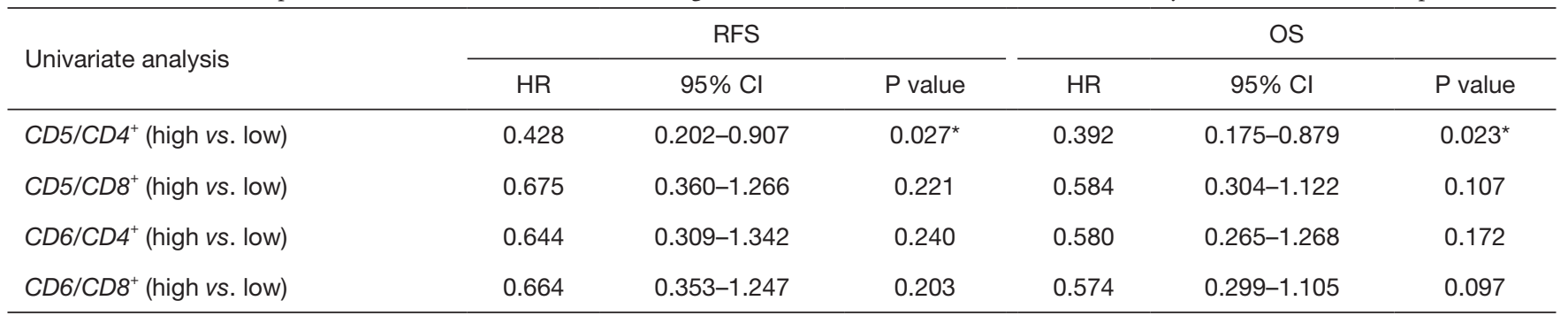

*, P value <0.05. NSCLC, non-small cell lung cancer; RFS, relapse-free survival; OS, overall survival; HR, hazard ratio; Cl, confidence interval.

Table S3 Survival analysis of $C D 5$ and $C D 6$ ratios in NSCLC patients of training cohort. Cox univariate analyses were conducted with dichotomized relative expression of $C D 5 / C D 3 D, C D 5 / C D 3 E, C D 5 / C D 4, C D 5 / C D 8, C D 6 / C D 3 D, C D 6 / C D 3 E, C D 6 / C D 4$ and $C D 6 / C D 8$ ratios in 186 patients

\begin{tabular}{|c|c|c|c|c|c|c|}
\hline Univariate analysis & \multicolumn{3}{|c|}{ RFS } & \multicolumn{3}{|c|}{ OS } \\
\hline$C D 5 / C D 3 D$ (high vs. low) & 1.010 & $0.687-1.485$ & 0.958 & 0.859 & $0.567-1.301$ & 0.473 \\
\hline$C D 5 / C D 3 E$ (high vs. low) & 0.925 & $0.631-1.357$ & 0.691 & 0.846 & $0.559-1.281$ & 0.430 \\
\hline CD5/CD4 (high vs. low) & 0.906 & $0.617-1.330$ & 0.613 & 0.633 & $0.406-0.988$ & $0.044^{*}$ \\
\hline$C D 6 / C D 3 D$ (high vs. low) & 1.093 & $0.744-1.605$ & 0.650 & 1.081 & $0.714-1.637$ & 0.712 \\
\hline$C D 6 / C D 3 E$ (high vs. low) & 0.977 & $0.664-1.436$ & 0.905 & 0.908 & $0.598-1.379$ & 0.650 \\
\hline $\mathrm{CD6} / \mathrm{CD} 4$ (high vs. low) & 0.858 & $0.585-1.259$ & 0.435 & 0.798 & $0.527-1.209$ & 0.287 \\
\hline$C D 6 / C D 8$ (high vs. low) & 0.970 & $0.661-1.424$ & 0.878 & 0.835 & $0.551-1.266$ & 0.396 \\
\hline
\end{tabular}

*, P value <0.05. NSCLC, non-small cell lung cancer; RFS, relapse-free survival; OS, overall survival; HR, hazard ratio; Cl, confidence interval. 